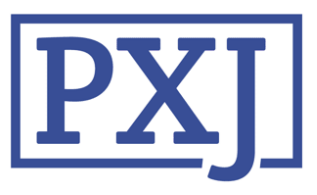

Patient Experience Journal

Volume 4 | Issue 3

Article 10

2017

\title{
Patient safety: just ask. Patients as reporters of real-time safety data; a pilot project to improve patient safety in secondary care
}

Thomas A. Cairns Dr

NHS

lain Mccallum $\mathrm{Mr}$

NHS

Follow this and additional works at: https://pxjournal.org/journal

Part of the Health and Medical Administration Commons, Health Policy Commons, Health Services Administration Commons, and the Health Services Research Commons

\section{Recommended Citation}

Cairns TA, Mccallum I. Patient safety: just ask. Patients as reporters of real-time safety data; a pilot project to improve patient safety in secondary care. Patient Experience Journal. 2017; 4(3):55-60. doi: 10.35680/2372-0247.1169.

This Research is brought to you for free and open access by Patient Experience Journal. It has been accepted for inclusion in Patient Experience Journal by an authorized editor of Patient Experience Journal. 
Patient safety: just ask. Patients as reporters of real-time safety data; a pilot project to improve patient safety in secondary care

\section{Cover Page Footnote}

Acknowledgements Dr Andrew McHattie, Dr Allan Burt, Dr Kian Dastoori, Dr Farah Hussain, Dr Kiran

Panesar, Mr John Wayman, Dr Jeremy Rushmer, 


\title{
Patient safety: just ask. Patients as reporters of real-time safety data; a pilot project to improve patient safety in secondary care
}

Thomas A.Cairns, NHS, tomacairns@gmail.com

Iain Mccallum, NHS, iainmccallum@nbs.net

\begin{abstract}
The Berwick review into patient safety recommended 'involving patients in the healthcare organisation and seeking out the patient voice as an essential asset to monitor safety.' (1) However routine data collection from patients in our institution is retrospective and doesn't focus on safety. Our objective was to create a patient-centred mechanism to monitor patient-perceived safety concerns and provide immediate resolution of highlighted issues. A pragmatic 6question questionnaire was developed containing 4 scored and 2 free text questions. This questionnaire was piloted and adjusted before being administered to all inpatients meeting the inclusion criteria in our institution on one day. Safety issues raised were triaged and acted upon according to an agreed protocol providing a mechanism for immediate resolution. 225 patients were inpatients in the clinical areas surveyed of which 149 were eligible and 148 participated (99\% participation). The majority (>95\%) felt nothing about their stay was unsafe and felt they had no concerns about their treatment plan. However multiple themes regarding patient safety were identified including environmental issues, staffing levels, supervision of vulnerable patients and handover of clinical information. None of the issues reported by patients had been reported through existing hospital incident reporting systems. Safety issues triaged as requiring immediate attention were fed back to appropriate teams on the day to allow immediate learning. These results suggest that patients find safety reporting of their care acceptable via a simple questionnaire. Integration of this new process may increase overall safety reporting and allow targeted improvements in safety, quality and patient experience.
\end{abstract}

\section{Keywords}

Patient engagement, National Health Service, patient-centred care, quality of care, patient safety, safety reporting, safety culture

\section{The Problem}

There is no current NHS-wide or local Trust-wide system in place to monitor and act upon real time inpatientperceived safety concerns. This is despite national leaders calling for trusts to put patients at the centre of a safety culture and seek their help in monitoring safety. Not monitoring patient-perceived safety concerns may lead to missed opportunities to improve the safety culture and safety environment within our institutions.

Organisations rely on staff reporting on safety incidents, however this alone may not represent all incidents that occur nor address patients safety concerns. Many trusts have trialled postal questionnaires to gain patient feedback post-discharge from hospital. However, these are largely focused on quality rather than specifically safety and have the disadvantage of providing retrospective data from which learning opportunities may have been lost. The creation of a new prospective process to gather this data may provide local opportunities to improve patient safety at a ward-based level and enhanced safety culture hospitalwide.

\section{Background}

The World Health Organisation (WHO), through its Patient Safety Programme, adopted as a priority the objective to facilitate and stimulate global learning through enhanced reporting of patient safety incidents. Worldwide, healthcare organisations have recognised both the ubiquitous nature of medical error and the rich learning opportunities available to learn from these. (2) Successful safety reporting mechanisms must promote an open culture of safety and return institutional learning from incidents to the relevant parties. UK institutions have largely relied on medical and nursing staff initiated, voluntary, incident-reporting systems. Such systems have several failures including variable definition of what constitutes inevitable harm vs. preventable harm, safety culture, perception of value from reporting and fear of blame. $(2,3)$ Current reporting systems led by clinicians only detect a minority of errors and an audit of prescribing errors found that only $0.12 \%$ of prescribing errors discovered at audit led to an incident report. Although around $22 \%$ of clinically important errors were detected by staff only around half of these were reported. (4). Data

Patient Experience Journal, Volume 4, Issue 3- 2017

(C) The Author(s), 2017. Published in association with The Beryl Institute and Patient Experience Institute

Downloaded from www.pxjournal.org 
suggest that fear of blame by colleagues, litigation and disciplinary action were important barriers in reporting errors. (5)

The Berwick review into patient safety in the UK recommended embracing patient safety, involving patients in the healthcare organisation and seeking out the patient voice as an essential asset to monitor safety. Patient reporting of high quality of care has been shown to correlate with low rates of preventable complications such as pressure ulcers. (6). Attempts have been made to create valid questionnaires for patient reporting of safety and quality, although to date these result in institutional data rather than individual data allowing immediate intervention as well as institutional learning. (7) Patient reporting of safety data has been shown to be accurate but a recent review has shown a paucity of reports in the literature. It suggests that the optimal reporting tool will mix 'scoring' type questions to monitor performance with open questions to aid specific reporting of incidents and address issues of differing terminologies and perceptions of safety of patients and healthcare professionals. (8) With the identified inadequacies of medical led safety reporting we sought to trial a simple, real-time, pragmatic, patient led survey to report general feelings on safety of care and specific concerns for escalation.

\section{Baseline levels of incident reporting}

The National Reporting and Learning System (NRLS) in the UK published an annual Patient Safety Incident Report in September 2014 for our NHS Trust. This is designed to compare incident reporting rates, types of incident, degree of harm and regularity of reporting in this trust in comparison to other similar medium size acute NHS trusts. The report showed that our organisation reports 6.8 incidents per 100 admissions (2,942 incidents in a 6-month period). This is lower than the median reporting rate of 7.82 for this group of 46 medium acute organisations, with 33 trust reporting more frequently. This illustrates that there is potential scope to increase incident reports within this trust in line with other similar acute medium size hospital trusts. Increased reporting has been associated with improved patient safety and an improved safety culture.

\section{Methods}

A 6-point safety questionnaire with open and closed questions was developed after reviewing of other available safety questionnaires in the literature, discussion with medical colleagues, the head of the patient experience department and patients. The questionnaire was reviewed and developed with guidance from four inpatients. (See Appendix)

This initial questionnaire was piloted on two surgical wards with the following aims: to assess feasibility of the planned delivery strategy; gauge acceptability to the patients; to optimise the questionnaire layout, language and clarity; and to assess if relevant issues were likely to be raised. The data gathered suggested that useful qualitative and quantitative data about safety and quality could be gained and that the performance plan was feasible with good acceptability to patients. No simple UK patient safety questionnaire was available at the time of this project; therefore a local questionnaire was developed.

Prior to rolling out on a complete patient sample an escalation algorithm was developed with the medical director to ensure that any time-critical serious patient safety issues would be effectively managed on the day of the project. This included same day feedback of immediate safety critical concerns with appropriate escalation through clinical management structures for serious problems. In addition, formal reporting of summative data from the project was planned at the Trust Wide Quality and Safety Board Meeting together with individual ward reports.

As this was a novel project to assess efficacy five junior doctors were recruited to help with data collection. Doctors distributing the survey were provided with written and verbal briefing outlining the aims of the project and methodology. They hand delivered the survey to patients providing a brief explanation to supplement written information contained in the survey (APPENDIX), answered queries and left the patient to complete the survey, collecting the completed form around 15 minutes later. Doctors did not survey patients who were under their own care to minimise influence on reporting and undertook this task in their own free time and were provided with a note of their participation for addition to their clinical portfolio.

All in-patients in Cumberland Infirmary on the morning of the survey were eligible to participate except those fulfilling exclusion criteria: $<18$ years of age, infective isolation, severe dementia, end-of-life care, critical care, recent general anaesthetic, receiving personal care, and those deemed too unwell to participate by nursing staff. No incentive was offered to patients to participate.

The survey was performed on a single morning across all adult wards in the Cumberland Infirmary (14 wards, 149 patients). Several wards were closed due to infection and so the total sample size was reduced compared to initial expectations. Analysis was in two phases: immediate analysis to identify time-critical safety issues mandating immediate feedback and remedial action and later thematic analysis of other issues reported. Grouping of reported issues into themes was done in a non-formal fashion by one investigator (TC), formal thematic analysis was not deemed necessary to achieve the aims of the project due to its exploratory nature. 


\section{Results}

There were 225 potentially eligible medical or surgical inpatients on the day of the survey of which 77 were excluded due to meeting the exclusion criteria. 149 were approached to complete the questionnaire, of which 148 patients chose to participate ( $99 \%$ uptake)

All results were reviewed on the morning and one identified time critical safety issue was dealt with on the day. The survey was well received by the ward staff and patients with an uptake rate of $99 \%$ in those included. Ward reports were produced allowing rapid feedback to the ward and for the medical director and the results were presented to the quality and safety board within 6 weeks.

The results of the scored questions showed that almost all patients $(95 \%)$ did not feel anything about their hospital stay was unsafe, $(97 \%)$ did not have any current safety concerns about their treatment or care plan and (97\%) felt their medical and nursing care was safe or extremely safe. Most patients (87\%) thought it would be extremely easy or easy for them to raise safety concerns to medical or nursing staff suggesting a positive open safety culture.

However, despite this lack of safety issues reflected in the scored questions the free text questions identified 46 specific concerns from patients. These were classified into themes: Shortage of staff; poor supervision of vulnerable patients; cluttered / too small environment for care; late response to call bells; poor communication of management \& results; inadequate numbers of toilets/showers; poor continuity of staff; specific incident.

Most of the reports above are probably more accurately described as describing care quality and only within the specific incident category were 4 incidents identified where actual harm or potential harm came to patients. These included; loss of an important pre-hospital investigation (ECG) relevant to the patient's clinical course; attempt by porters to take a patient for a CT scan twice potentially exposing the patient to further radiation; an unmarked wet floor with risk of trips and injury; inadequate handover of complex medical history between medical teams resulting in poor care and frustration for the patient. The other 2 reported incidents by patients related to a late-night bed move to accommodate a patient in a single room and the physical examination of a patient without appropriate introductions or a chaperone.

The impact of how implementation of this survey could alter incident reporting was measured by analysis of the staff reporting system. Data was retrieved from the webbased reporting system (Ulysses) used by the trust. 443 incidents were reported within 2 weeks of the survey date (30/12/14-26/1/15). Analysis of these incidents showed that none of the specific incidents reported by the patient survey were reported by staff. There were no staff reports identifying low levels of ward nursing staff or issues relating to space as a problem in the data period identified. This suggests that the approach of engaging patients in safety reporting would increase the overall reporting of this institution with the potential for improving overall safety. Integration of this process into the trust has the potential to do this

\section{Discussion}

We aimed to seek out the patient voice as an asset in monitoring the safety and quality of care. Such an approach appears to be acceptable to patients with $99 \%$ of eligible patients participating. Overall patients were satisfied with the safety of their care with over $95 \%$ stating they did not feel anything about their hospital stay was unsafe and that they did not have any current safety concerns about their treatment or care plan. A minority of patients did identify safety concerns that were predominantly clustered around staffing levels, inadequate supervision of vulnerable patients, environmental factors and information availability to the responsible medical team.

Data available to date suggests that patients report differently depending on the type of question they are asked and so we endeavoured to ask open questions. This seems to be reflected in the responses we received which identified many factors not reported via existing staff led systems such as environmental and ergonomics issues. This may suggest that patients are useful observers of how the environment may influence safety issues. Staff reporting data would suggest that this is not an area that is deemed important to staff perhaps due to a perception of inevitability around the fashion in which the workplace is designed.

A distinct advantage of our approach was immediate escalation and resolution of specific incidents such as an incident raised regarding the examination of a patient without consent or introduction. This approach allowed early resolution of incidents with feedback to patients. There are obvious advantages in such an approach in terms of improvement in quality of care and safety culture. However, it does rely on a person with appropriate experience to triage and escalate patient reports as well as significant manpower which may limit the scalability of this exact approach. This project was undertaken with the full support of the Medical Director using a predefined protocol, empowering the triaging doctor to escalate the incident within, and if necessary, above the immediate clinical team caring for the patient until satisfactory resolution was achieved. Although only a small number of such incidents were identified these were all able to be resolved within the immediate clinical team. 
We found no concordance when we conducted a comparison of patient reported data with staff reported data by the existing safety reporting system used normally within the trust. Although this may initially seem surprising other reports have shown low concordance with less than $8 \%$ when examining only medication errors and no concordance when a similar open ended approach was used (9). However, our data does fit with existing evidence in this area where reports by patients are generally lesser in terms of preventability and severity. (7) This suggests that patient reporting may be an important method to overcome some of the existing limitations in staff led reporting systems.

\section{Conclusion}

What this project illustrates is that patients are keen and insightful observers of their clinical environment and this process has achieved its aim in creating an acceptable and welcome method of providing patients with an opportunity to voice their safety concerns.

\section{Future direction}

Longitudinal collection of such data would enable an institution to have a broader evaluation of both safety and quality factors focusing on a patient-centred approach. It is hoped that the trust will continue to repeat this project in the future and try to embed the process into the local safety reporting culture. Sustainability is achievable due to the short run time of the project within a morning and the ability to produce the results within a short time frame, although the current methodology does require significant manpower input. Success of this process could be established by monitoring for any increase in the number of incidents reported and in any positive changes seen in safety culture using the annual staff safety culture questionnaire. Sustainability of this type of project requires ongoing engagement by the management which is why the Medical Director was involved after inception of this project. Although the results within this institution are not transferable to other healthcare settings the concept of real time safety reporting from patients is entirely transferable. Further exploration of this concept should continue NHSwide, with development and optimisation of the process and testing within different hospitals with variable thresholds of reporting and safety culture.

\section{References}

1. A promise to learn- a commitment to act; Improving the Safety of Patients in England, National Advisory Group on the Safety of Patients in England, August 2013

2. Larizgoitia I, Bouesseau MC, Kelley E. WHO Efforts to Promote Reporting of Adverse Events and Global Learning. J Public Health Res. 2013 1;2(3):e29

3. Pronovost PJ, Morlock LL, Sexton JB, et al. Improving the Value of Patient Safety Reporting Systems in 'Advances in Patient Safety: New Directions and Alternative Approaches (Vol. 1: Assessment)'. Eds Henriksen K, Battles JB, Keyes MA, et al. Rockville (MD): Agency for Healthcare Research and Quality; 2008

4. Westbrook JI, Li L, Lehnbom EC, et al. What are incident reports telling us? A comparative study at two Australian hospitals of medication errors identified at audit, detected by staff and reported to an incident system. Int J Qual Health Care. 2015;27(1):1-9

5. Heard GC, Sanderson PM, Thomas RD. Barriers to adverse event and error reporting in anesthesia. Anesth Analg. 2012;114(3):604-14

6. Isaac T, Zaslavsky AM, Cleary PD, Landon BE. The relationship between patients' perception of care and measures of hospital quality and safety. Health Serv Res. 2010;45(4):1024-40

7. Bjertnaes OA, Sjetne IS, Iversen HH. Overall patient satisfaction with hospitals: effects of patient-reported experiences and fulfilment of expectations. BMJ Qual Saf. 2012;21(1):39-46

8. King A, Daniels J, Lim J, Cochrane DD, Taylor A, Ansermino JM. Time to listen: a review of methods to solicit patient reports of adverse events. Qual Saf Health Care. 2010;19(2):148-57

9. Weingart SN, Pagovich O, Sands DZ, et al. What can hospitalised patients tell us about adverse events? Learning from patient-reported incidents. J Gen Intern Med 2005;20:830e6 


\section{Appendix 1. Patient Safety Questionnaire}

The safety of patients is a priority in the NHS and our hospital.

Safety means avoiding mistakes or accidents relating to your care that may make your experience in hospital worse or cause you harm.

We would welcome your views on how safe you feel your care has been.

For each question please tick the box that most closely matches your experience.

Q1. Do you feel anything about your hospital stay has been unsafe? Yes No

If yes, what happened?

Q2. Do you have any current safety concerns about your treatment or care plan? Yes No

If yes, what concerns do you have?

Q3. How safe does your medical and nursing care feel?

Extremely safe

Safe

Neither safe or unsafe

Unsafe

Extremely unsafe

Don't know

Q4. How easy would it be for you to raise your concerns to medical or nursing staff if you thought something was unsafe with your care?

Extremely Easy

Easy

Neither easy or difficult

Difficult

Very difficult

Don't know

What is the reason for the response you have given?

Q5. What improvements, if any, would make you feel safer on this ward?

Q6. Please give examples of any safety issues you have seen on this ward? 
Appendix 2. Escalation Plan for Safety Issues

\section{ESCALATION PLAN FOR SAFETY ISSUES}

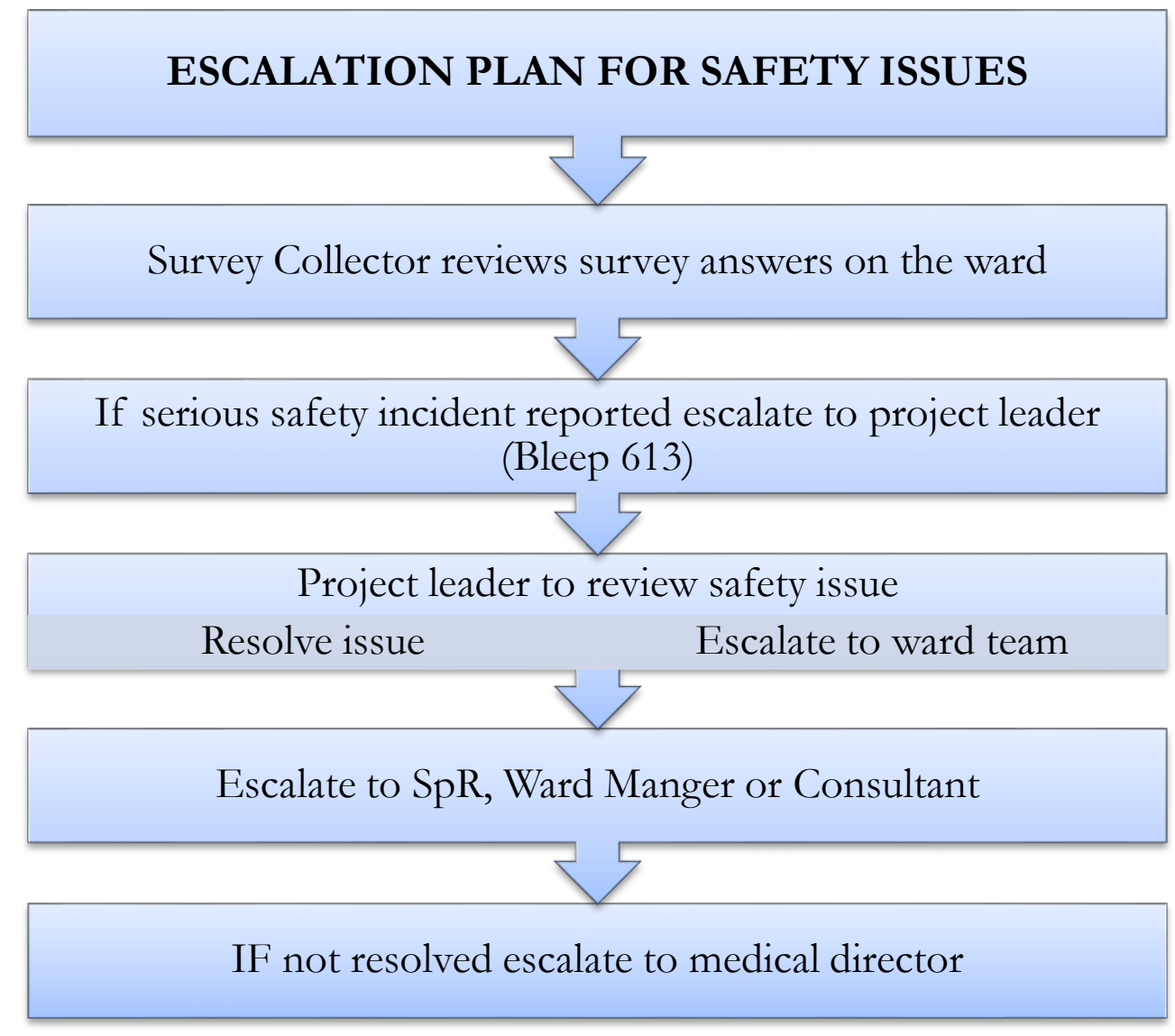

\begin{tabular}{|c|c|}
\hline Ward & \\
\hline Bed number & \\
\hline Male/Female & \\
\hline Age & \\
\hline Complete & \\
\hline Reviewed & \\
\hline
\end{tabular}

